\section{Morphological Characterization of Turf-type Tall Fescue Genotypes}

Eric Watkins ${ }^{1}$ and William A. Meyer ${ }^{2}$

Department of Plant Biology and Pathology, Rutgers University, 59 Dudley Road, Foran Hall, New Brunswick, NJ 08901

Additional index words. Festuca arundinacea, turfgrass breeding, Rhizoctonia solani, seed production

Abstract. Recently, turfgrass breeders have developed many improved turf-type tall fescue (Festuca arundinacea Schreb.) cultivars. Due to the large number of cultivars currently available to turfgrass managers and researchers, we have classified turf-type tall fescue cultivars into six groups based primarily on several morphological measurements. This type of classification is important for turfgrass breeders because many breeding decisions are made based on observations in a spaced-plant nursery. The major objective of this study was to classify tall fescue cultivars and selections based on spaced-plant measurements and to then compare those results with turf performance. A spaced-plant nursery consisting of 36 cultivars and selections was established in September 1998 at Adelphia, N.J. Plant height, panicle length, flag leaf width and length, subtending leaf width and length, and subtending internode length were measured 10 days after anthesis in 1999 and 2000 . Additionally, a turf trial was established at North Brunswick, N.J., that included the same 36 cultivars and selections. The turf plots were evaluated for several traits including overall turfgrass quality, density, and susceptibility to brown patch disease. Based on principal component analysis of morphological measurements, along with turf trial data, all cultivars and selections were assigned to one of six groups: forage, early-standard, standard, early semi-dwarf, semi-dwarf, and dwarf. In turf plots, the semi-dwarf, early-semi dwarf, and dwarf groups were the topperforming types in terms of overall turfgrass quality, and the forage and early-standard cultivars had the lowest overall quality ratings. The dwarf types did not perform well under summer stress, especially in terms of brown patch disease incidence. The results of this study suggest that when developing cultivars for higher maintenance situations, turf-type tall fescue breeders should focus on the development of semi-dwarf cultivars.

Turf-type tall fescue cultivar development has progressed rapidly. Tall fescue is used in many different situations including home lawns, athletic fields, golf course roughs, and low maintenance areas. Recently-developed cultivars consistently outperform older cultivars such as 'Falcon' (Shearman and Morris, 1996). In the 1940s, 'Kentucky-31'became the first tall fescue cultivar to be used as a turfgrass (Fergus and Buckner, 1972). The firstimproved turf-type tall fescue, 'Rebel', was developed by C.R. Funk in 1981 (Funk et al., 1981). From that point on, gradual improvement has been made in regard to growth habit, insect resistance, mowability, leaf texture, color, drought tolerance, and turf density (Morris, 2001). Currently, a large number of tall fescue cultivars are being sold for use as turf; the 2001 National Turfgrass Evaluation Program National Tall Fescue Test, which includes most available commercial cultivars, consists of 160 entries. As the number of private and public tall fescue breeders continues to increase, the number of available cultivars will also continue

Received for publication 4 Feb. 2003. Accepted for publication 31 Aug. 2003. Research supported by the Rutgers Center for Turfgrass Science, New Jersey Agricultural Station, and New Jersey Turfgrass Association Publication No. D-12180-3-03. Thank you to everyone on the turf breeding crew for helping take measurements. We also thank Stacy Bonos for helping with statistical analysis.

${ }^{1}$ Graduate research assistant; e-mail ericw@ rci. rutgers.edu.

${ }^{2}$ Professor.

to increase. These cultivars represent a wide range of morphology, especially when planted in a breeding nursery. Limited research has been done looking at the morphological differences that exist between the many turf-type tall fescue cultivars.

Grouping existing cultivars into groups based on morphology would be an aid to both researchers and plant breeders. Tall fescue cultivars have been somewhat characterized using turf trial data (Fermanian et al., 1996). The advantages and disadvantages of tall fescue cultivars with a dwarf growth habit have also been discussed (Hurley, 1990; Meyer and Bonos, 2001).

Substantial phenotypic and genotypic variation exists between many turf-type tall fescue cultivars. The source of most current tall fescue germplasm consists of plants introduced to the United States in the late 1800s. These plants were later the source of collections in old turf areas throughout the northeastern and southeastern U.S. made from 1962-82 (Funk and Meyer, 2001). Unlike other cool-season turfgrass species, tall fescue collection efforts in Europe and Asia have not been very effective; therefore, very little new germplasm has been added to the turf-type tall fescue germplasm pool. Nevertheless, plant breeders have been able to exploit cross-pollination in the species, creating genotypic and phenotypic variability.

Turfgrass breeders make many of their selections in large spaced-plant nurseries. Breeding decisions are often based on characteristics that are not seen in a turf plot, therefore, the plant breeder does not know if a plant has good turfgrass characteristics for at least a year after initial selection in the breeding nursery. The ability to identify additional morphological traits in unmowed spaced plants that correlate with improved turf performance would be a valuable asset to the plant breeder. Some of the primary characteristics of a superior turf-type tall fescue cultivar are good turf density, fine leaf texture, dark green color, and resistance to brown patch disease caused by Rhizoctonia solani Kühn.

The primary purpose of this study was to group current tall fescue germplasm into morphological groups based on field measurements and to then compare characteristics of the various groups in mowed turf plots. A second objective of this study was to evaluate the various tal fescue types for susceptibility to brown patch disease in turf plots so that turfgrass breeders can identify traits in a spaced-plant nursery that could lead to a reduction in disease incidence.

\section{Materials and Methods}

Spaced-plant study. In September 1998, tall fescue plants representing 36 cultivars and selections (Table 1) were established in a greenhouse. These plants were established

Table 1. Tall fescue cultivars and selections evaluated in a spaced-plant nursery established in Octobe 1998, grouped by type.

Forage

Kentucky-31

Early standard

Falcon

Standard

Coronado Gold

Falcon II

Jaguar 3

Rebel Jr.

Rebel 3D

Tarheel

Wolfpack

Semi-dwarf

Apache II

FFT

Finesse

Masterpiece

Millennium

Picasso

Mustang 3

Plantation

Rebel Sentry

Rembrandt

Scorpion

Shortstop II

SR 8250

Adam's Valley

5301

$5301 \mathrm{E}+$

$5301 \mathrm{E}-$

Early semi-dwarf

DLSD

Endeavor

Empress

Prospect

Dwarf

DDL-97

DWP

Matador

Pick FA N-93

5DH-97

8001 


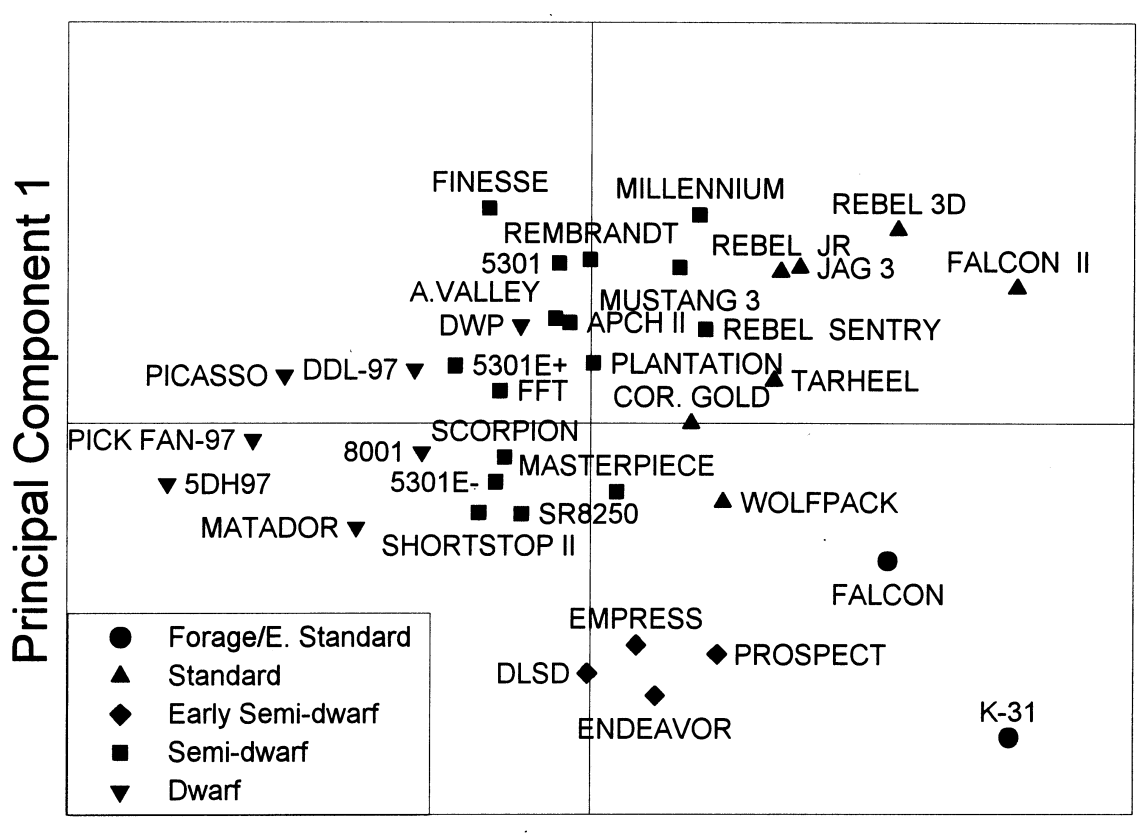

Principal Component 2

Fig. 1. Principal component analysis of tall fescue cultivars and selections based on morphological characteristics in 1999. PC1 accounted for $80.8 \%$ of the variation and correlated with panicle length, flag leaf length, subtending leaf length, plant height, subtending leaf width, and flag leaf width. PC2 accounted for $10.9 \%$ of the variation and correlated with heading date.

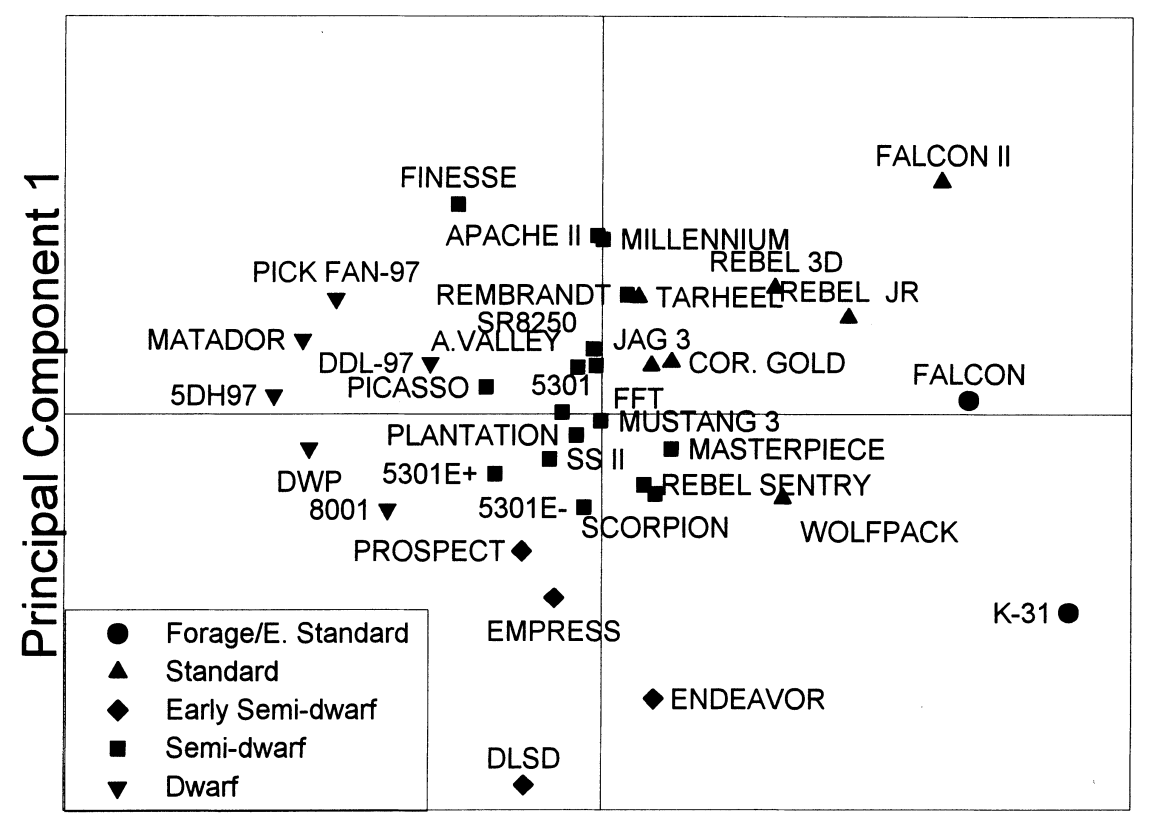

Principal Component 2

Fig. 2. Principal component analysis of tall fescue cultivars and selections based on morphological characteristics in 2000. PC1 accounted for $67.5 \%$ of the variation and correlated with plant height, subtending internode length, panicle length, flag leaf length, and flag leaf width. PC2 accounted for $19.4 \%$ of the variation and correlated with heading date and flag leaf width

in a nursery on 23 Oct. 1998 . The study consisted of five replications with each replication containing 11 plants from each cultivar or selection. The study was established on a Freehold sandy loam (fine, mixed, mesic, Typic Hapludult) and plants were spaced $0.6 \mathrm{~m}$ apart. The test received $7.3 \mathrm{~g} \cdot \mathrm{m}^{-2}$ of $\mathrm{N}$

(34N-0P-0K) in October 1998, March 1999, and April 1999. Morphological measurements were taken in both 1999 and 2000. Plants were not mowed until after measurements were taken in both years.

Morphological measurements were made $\approx 10 \mathrm{~d}$ after anthesis. Heading date was deter- mined as the day when the first five seed heads were completely emerged and was recorded as days after 1 Apr. of each year. Plant height was measured from the base of the crown to top of panicle (extremely tall panicles were avoided). Panicle length of three panicles per plant was measured from flag leaf to top of panicle. Length and width of three flag leaves per plant were measured, as well as the length and width of three subtending leaves per plant. Length of the first internode subtending the flag leaf was measured on three stems per plant.

Turf plots. A turf trial was established in Sept. 1998 in North Brunswick, N.J., on a Nixon loam (fine-loamy, mixed, mesic Typic Hapludult). The trial consisted of 36 cultivars and selections replicated three times in a randomized complete block design. Plots were $1.83 \times 1.22 \mathrm{~m}$ and seeded at a rate of $17.9 \mathrm{~g} \cdot \mathrm{m}^{-2}$. Plots received $\approx 17.0 \mathrm{~g} \cdot \mathrm{m}^{-2}$ of $\mathrm{N}$ $(16 \mathrm{~N}-1.8 \mathrm{P}-6.7 \mathrm{~K})$ per growing season. In the spring and fall, $\approx 7.3 \mathrm{~g} \cdot \mathrm{m}^{-2}$ of $\mathrm{N}(16 \mathrm{~N}-1.8 \mathrm{P}-$ $6.7 \mathrm{~K}$ ) was applied. In July and August of both years, the turf plots were fertilized (2.4 $\mathrm{g} \cdot \mathrm{m}^{-2}$ of $\left.\mathrm{N}(16 \mathrm{~N}-1.8 \mathrm{P}-6.7 \mathrm{~K})\right)$ to encourage the development of brown patch. Plots were mowed at $3.8 \mathrm{~cm}$ with a reel mower as needed and clippings were returned. During the summer, supplemental irrigation was used only to avoid severe drought stress.

To evaluate for susceptibility to brown patch disease, the turf plots were inoculated in June 1999 to create uniform disease pressure. Rhizoctonia solani isolates were grown on onehalf strength potato dextrose agar. After the isolates had grown enough to cover the entire surface of the agar, the agar was cut into small pieces $\left(\approx 1.0 \mathrm{~cm}^{2}\right)$. About one-half of each plate of each isolate was added to an Erlenmeyer flask containing $250 \mathrm{~g}$ of sterilized kentucky bluegrass (Poa pratensis L.) seed and $100 \mathrm{~mL}$ of distilled water. The fungus was allowed to grow on the seed for $\approx 2$ weeks. The seed was removed from the flasks and air-dried. After the inoculum was fully dried, the inoculum was sieved to uniform consistency (No. 13T ( $0.12 \times$ $0.95 \mathrm{~cm}$ ) Seedburo Equipment Co.) and spread on the turf plots using a drop spreader at a rate of $3.2 \mathrm{~g} \cdot \mathrm{m}^{-2}$. After inoculation, the plots were irrigated with $\approx 0.64 \mathrm{~cm}$ of water.

Turf data was collected during 1999 and 2000. Ratings were taken for several turf characteristics including overall turfgrass quality and severity of brown patch disease. Ratings for these characteristics were taken visually using a 1 to 9 scale $(9=$ best turfgrass quality, $9=$ least brown patch disease). In 1999, tiller counts were taken in order to quantify turf density. Total number of tillers was counted from two plugs $\left(0.01 \mathrm{~m}^{2}\right.$ per plug) per plot.

Data analysis. The relationship between tall fescue cultivars and selections based on morphological characteristics was tested by subjecting the data from the spaced-plant nursery to multivariate PCA PROCPRINCOM (SAS Institute, 1985). This type of analysis was used by Bonos et al. (2000) to confirm kentucky bluegrass genotype classifications. The principal component analysis, when plotted against the major principal components, can reveal clusters of morphologically similar 
phenotypes (Iezzoni and Pritts, 1991). In our study, 36 cultivars and selections were used for the analysis. For 1999 data, six morphological variables were used for the analysis and for 2000 data, seven morphological variables were used.

Principal component analysis restructures the entire data set of variables (in this study, the morphological measurements) into smaller sets of components of the original variables. These components can then be scored against major principal components (Iezzoni and Pritts, 1991). The original data are transformed to create new variables and axes that account for more variation in the original data than any single variable alone. The first transformation (PC1) accounts for as much of the variation as possible and represents the major axis. A second transformation ( $\mathrm{PC} 2)$ is performed which accounts for as much of the remaining variation as possible. This continues until the total number of transformations equals the number of original variables (in this case, six in 1999 and seven in 2000). This process is further described by Iezzoni and Pritts (1991).

Eigenvalues, (the total variance accounted for by each equation) that are greater than 1.0 should contain more information than any other single variable alone. These eigenvalues $(>1.0)$ should be used in data interpretation (Iezzoni and Pritts, 1991).

Principal component analysis was performed on data from each year separately and the two major principal components (PC1 and PC2) were plotted against each other for each year (Figs. 1 and 2). Cultivars and selections were assigned to morphological groups primarily based on these plots; however, other factors such as recent turf plot data, field observation, and a cultivar's breeding history were sometimes used to determine groupings. Cultivars that clustered together in both years were assigned to the same morphological group. In cases where cultivars fell between two groups, the aforementioned factors were used to determine groupings. Data for each entry were combined to develop means for each type (Table 3 ) and subjected to analysis of variance (ANOVA). The forage and early standard groups were combined for analysis. Means for each group were separated using Tukey's means separation. Turf plot data were also analyzed by using ANOVA and Tukey's means separation according to the type group assignments from morphological measurements and principle component analysis (PCA) of the spaced-plant study. The same 36 cultivars and selections were used in both the morphological measurements study and the turf trial.

\section{Results and Discussion}

Because turf-type tall fescue was developed from forage tall fescue, many years of intensive breeding were needed to develop acceptable turf quality. The most important component of turf quality that has been improved in tall fescue is turf density. Compared to earlier-developed cultivars such as Falcon and Rebel, most of the newer cultivars have superior density. This increased density has been primarily due to the selection of plants that exhibit narrow leaves in spaced-plant nurseries. The major drawback to increased density is that many of these newe cultivars are more susceptible to brown patch than the earlier-developed cultivars (Watkins et al., 2002).

To select superior tall fescue genotypes, the different types of tall fescue and how they relate to turf performance must be understood. Based on principal component analysis of morphological variables, available data, field observation, and breeding histories, we have classified current turf-type tall fescue cultivars into six categories: forage, early standard, standard, early semi-dwarf, semi-dwarf, and dwarf.

Spaced-plant study. All morphological measurements were smaller in 1999 compared to 2000 because the plants were planted in the nursery late in the year in 1998 and did not have sufficient time for growth; however, the differences did not affect the analysis. Plotting $\mathrm{PC} 1$ vs. PC2 resulted in clustering of the entries in both years (Figs. 1 and 2). In 1999, PC1 was best correlated with (listed in decreasing order) panicle length, subtending leaf length, flag leaf length, plant height, subtending leaf width, and flag leaf width. In 2000, PC1 was best correlated with (listed in decreasing order) plant height, subtending internode length, panicle length, flag leaf length, and flag leaf width. PC2 was best correlated with heading date in 1999 and heading date and flag leaf width in 2000 (Table 2)

The forage group consists of Kentucky-31 and other cultivars with similar characteristics.

Table 2. Eigenvectors of the principal component axes (PC) from principal component analysis of tall fescue cultivars and selections. Eigenvalues and their contribution to total variation are listed.

\begin{tabular}{|c|c|c|c|c|c|c|c|c|c|c|c|c|c|c|}
\hline \multirow{2}{*}{ Character } & \multicolumn{14}{|c|}{ Eigenvectors } \\
\hline & \multicolumn{2}{|c|}{$\mathrm{PC} 1$} & \multicolumn{2}{|c|}{$\mathrm{PC} 2$} & \multicolumn{2}{|c|}{ PC3 } & \multicolumn{2}{|c|}{$\mathrm{PC} 4$} & \multicolumn{2}{|c|}{ PC5 } & \multicolumn{2}{|c|}{ PC6 } & \multicolumn{2}{|c|}{ PC7 } \\
\hline Heading date & -0.25 & -0.21 & 0.90 & 0.76 & 0.25 & 0.61 & 0.24 & -0.04 & 0.02 & 0.01 & 0.04 & 0.08 & 0.07 & --- \\
\hline Panicle length & 0.41 & 0.46 & -0.02 & -0.06 & 0.29 & 0.25 & 0.10 & 0.57 & 0.51 & 0.63 & 0.67 & 0.03 & -0.18 & --- \\
\hline Flag leaf length & 0.40 & 0.46 & 0.22 & 0.13 & 0.25 & -0.08 & -0.43 & -0.78 & -0.45 & 0.40 & -0.09 & 0.07 & -0.57 & --- \\
\hline Flag leaf width & 0.37 & 0.29 & 0.24 & 0.63 & -0.64 & -0.65 & 0.05 & 0.27 & -0.38 & -0.13 & 0.42 & -0.07 & 0.28 & --- \\
\hline Subtending leaf length & 0.40 & --- & 0.07 & --- & 0.35 & --- & -0.38 & --- & 0.08 & --- & -0.22 & --- & 0.71 & --- \\
\hline Eigenvalue & 5.65 & 4.05 & 0.76 & 1.17 & 0.37 & 0.42 & 0.13 & 0.20 & 0.04 & 0.11 & 0.03 & 0.05 & 0.01 & --- \\
\hline$\%$ of total variation & 80.8 & 67.5 & 10.9 & 19.4 & 5.2 & 7.1 & 1.8 & 3.3 & 0.6 & 1.9 & 0.4 & 0.8 & 0.2 & --- \\
\hline
\end{tabular}

Table 3. Heading date, plant height, panicle length, flag leaf length, and subtending internode length of tall fescue types planted in a spaced plant nursery in Sept. 1998.

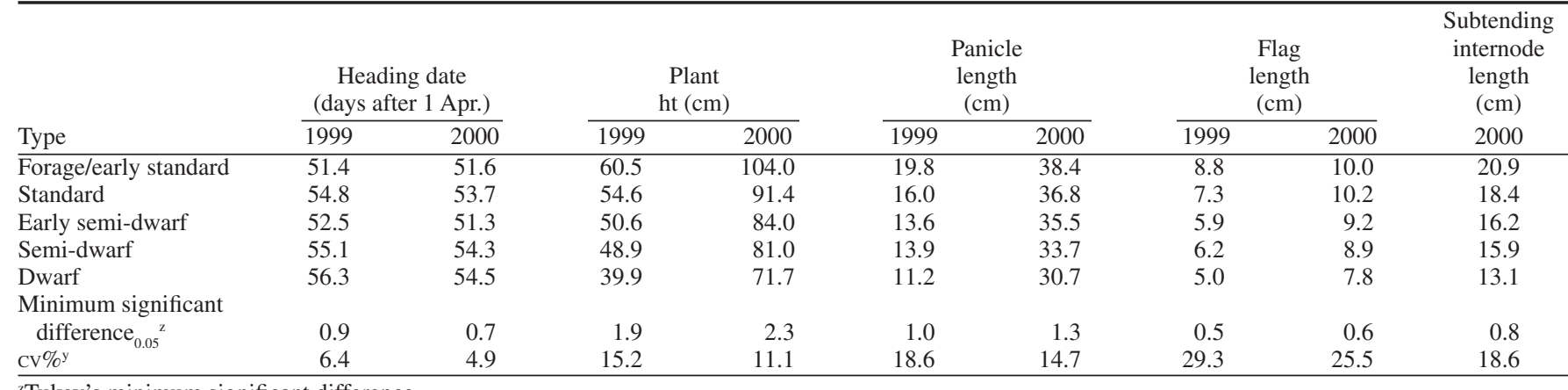

's minimum significant

${ }^{\mathrm{y}}$ The coefficient of variability $(\mathrm{Cv})$ is the total $\mathrm{cv}$ for the analysis of variance. 
Table 4. Performance of tall fescue types in a turf trial seeded in Sept. 1998.

\begin{tabular}{lccccc}
\hline & Turf quality $^{\mathrm{z}}$ & \multicolumn{3}{c}{ Brown patch $^{\mathrm{y}}$} & Tiller no. \\
\cline { 3 - 6 } Type & 1999 & 2000 & $1999^{\mathrm{x}}$ & $2000^{\mathrm{w}}$ & 1999 \\
\hline Semi-dwarf & 5.7 & 6.1 & 3.5 & 5.3 & 108.5 \\
Early semi-dwarf & 5.7 & 6.0 & 4.0 & 5.0 & 106.6 \\
Dwarf & 5.2 & 6.0 & 2.3 & 4.5 & 110.8 \\
Standard & 4.9 & 4.4 & 4.0 & 5.0 & 101.0 \\
Forage/early standard & 2.2 & 1.3 & 6.0 & 4.7 & 74.1 \\
Minimum significant difference $_{0.05}{ }^{\mathrm{u}}$ & 0.6 & 0.9 & 1.4 & 1.6 & 15.8 \\
\hline
\end{tabular}

${ }^{\mathrm{z}} 1$ to 9 scale, 9 = best turf quality. The 1999 and 2000 data are averages of 13 and 9 rating dates, respectively.

y 1 to 9 scale, 9 = least brown patch disease.

${ }^{\times}$Rating taken 9 Aug. 1999

${ }^{\text {w}}$ Average of two rating dates taken 8 and 15 Aug.

"Tiller number determined by averaging number of tillers in two samples $\left(91.5 \mathrm{~cm}^{2}\right.$ each $)$ per plot taken 9 Sept. 1999.

uTukey's minimum significant difference.

The primary advantage that is found in this group is increased seed production. The high productivity of Kentucky-31 is a major reason that it continues to be sold in large quantities even though it has the lowest turfgrass quality of any commercial cultivar (Watkins et al., 2003). Kentucky-31 is the earliest maturing of the cultivars in this study and also grows to be the largest plant in a spaced plant nursery. The early standard group includes cultivars such as Falcon and 'Olympic'. These plants are similar morphologically to the forage group in a spaced plant nursery. This group also has a very early maturity and high seed yields. The forage and early standard groups are easy to identify in breeding nurseries and were consistently separated with PCA in both years (Figs. 1 and 2).

Standard cultivars include 'Rebel Jr.', 'Falcon II' and 'Coronado Gold'. The standard type is characterized by a medium maturity and this type of tall fescue can become quite large in a spaced plant nursery. Plants of this type are greatly reduced in size compared to both the forage and early standard groups (Table 3 ).

The semi-dwarf group consists of a majority of the cultivars that have been developed in more recent years. These plants have a medium height, medium maturity, and finer leaves than the previous three groups (Table 3). Some examples of this group include 'Millennium', 'Rembrandt', and 'Plantation'.

The early semi-dwarf group has the same characteristics as the semi-dwarf group with the exception of having earlier maturity. In our study, the early semi-dwarf plants had heading dates that were, on average, 2.6 to 3.0 d earlier than the semi-dwarf group (Table 3). This trait can be very important to seed growers in the Pacific Northwest because early-maturing cultivars can complete seed production prior to severe summer stress from disease, heat, and drought. The heading dates for the early semidwarf type were earlier than any other group except for the forage/early standard cultivars (Table 3). Breeding efforts have been able to maintain high turf quality in these cultivars and selections (Table 4) while selecting for earlier anthesis. 'Prospect', 'Empress', and 'Endeavor' are cultivars belonging to this group.

Dwarf-type tall fescue cultivars have narrow leaves and the highest turf density of any of the tall fescue groups. As expected, plant height measurements for the dwarf group were much reduced compared to all other groups (Table 3 ). This group includes the cultivar 'Matador' and several experimental lines.

Turf plots. In turf plots, the groups differed significantly in overall turf quality (semidwarf, early semi-dwarf, dwarf $>$ standard $>$ forage/early standard) (Table 4). Although statistically insignificant, noticeable differences were also observed in resistance to brown patch with the dwarf type consistently ranking last (Table 4 ).

The most difficult trait to develop in tall fescue, to this point, has been resistance to brown patch disease. There are currently no turf-type tall fescue cultivars that are completely resistant to brown patch (Watkins et al., 2000, 2002). Turf density plays an important role in brown patch severity (Giesler et al., 1996a, 1996b). Because of the lack of brown patch resistance in tall fescue germplasm, plant breeders should select plants having traits that do not result in extremely high density when planted as a turf. Turf managers may be able to reduce the impact of brown patch by mixing tall fescue with kentucky bluegrass (Dunn

The forage group shows less susceptibility to brown patch, which may be due partly to wider leaves (Green et al., 1999) or lower turf density (Giesler et al., 1996a, 1996b) (Table 4). A negative result of decreased density is greater invasion by weedy species. A major advantage of Kentucky-31 is that it can withstand drought conditions longer than most other turf-type cultivars. Bowman and Macaulay (1991) found that Kentucky-31 used more soil water before wilting than other turf-type tall fescue cultivars under mowed field conditions.

The turf quality of early-standard cultivars is improved over the forage group. The density is not sufficient for most turf situations and these cultivars exhibit an unattractive color. Cultivars from these two groups are better able to withstand severe drought stress than other turf-type cultivars

Standard cultivars are characterized by average turf quality and density. These cultivars can be quite successful when used in low maintenance situations, especially when supplemental irrigation is not possible. 'Wolfpack' is able to maintain bright green color when many other cultivars begin to show wilting from drought (Watkins et al., 2000).

The semi-dwarf group contains many of the et al., 2002). top performing turf-type tall fescue cultivars. This group has above-average turf density and excellent turf quality. In 1999, semi-dwarf cultivars and selections had an average quality of 5.7 ( 1 to 9 scale, $9=$ best turfgrass quality), and in 2000 , the average quality of the group was 6.1 . These ratings suggest very good turfgrass quality. The early semi-dwarf group shows similar turf characteristics to the semi-dwarf group. The overall turfgrass quality averages of these groups were not significantly different in either 1999 or 2000.

Dwarf-type tall fescue cultivars have the highest turf density of any of the tall fescue groups (Table 4). Many recent selections, when grown under high maintenance conditions, look very similar to improved kentucky bluegrass cultivars. Unfortunately, this increased turf density can lead to increased susceptibility to brown patch and other diseases. In both 1999 and 2000, dwarf-type cultivars and selections had the lowest brown patch resistance of all the groups. Additionally, many cultivars and selections in this group perform poorly under heat stress. The advantage of this group is high turf quality when not affected by brown patch or other summer stresses. Dwarf-type tall fescue plants can be easily identified in the breeding nursery and should be avoided in breeding programs for the aforementioned reasons.

Based on our research, we suggest that turf-type tall fescue breeders should focus on selecting plants with a semi-dwarf growth habit. Combined with potential future advances in brown patch resistance, semi-dwarf tall fescue plants should produce the best overall, long-term turfgrass quality.

\section{Literature Cited}

Bonos, S.A., W.A. Meyer, and J.A. Murphy. 2000. Classification of kentucky bluegrass genotypes grown as spaced-plants. HortScience 35(5):910-913

Bowman, D.C. and L. Macaulay. 1991. Comparative evapotranspiration rates of tall fescue cultivars. HortScience 26(2):122-123.

Dunn, J.H., E.H. Ervin, and B.S. Fresenburg. 2002 Turf performance of mixtures and blends of tall fescue, kentucky bluegrass, and perennial ryegrass. HortScience 37(1):214-217.

Fergus, E.N. and R.C. Buckner. 1972. Registration of 'Kentucky-31' tall fescue. (Reg. No. 7). Crop Sci. 12:714.

Fermanian, T.W., J.E. Haley, K. Wessels, H.T. Wilkinson, and S. Han. 1996. Characterization of tall fescue and perennial ryegrass cultivars. J. Turfgrass Mgt. 1(4):63-79.

Funk, C.R., W.K. Dickson, and R.H. Hurley. 1981. Registration of 'Rebel' tall fescue. Crop Sci. 21:632.

Funk, C.R. and W.A. Meyer. 2001. Seventy years of turfgrass improvement at the New Jersey Agriculture Experiment Station. Proc. 10thAnnu. Rutgers Turfgrass Symp. p. 11-19.

Giesler, L.J., G.Y. Yuen, and G.L. Horst. 1996a. Tall fescue canopy density effects on brown patch disease. Plant Dis. 80:384-388.

Giesler, L.J., G.Y. Yuen, and G.L. Horst. 1996b. The microclimate in tall fescue turf as affected by canopy density and its influence on brown patch disease. Plant Dis. 80:389-394.

Green II, D.E., L.L. Burpee, and K.L. Stevenson. 1999. Components of resistance to Rhizoctonia solani associated with two tall fescue cultivars. 
Plant Dis. 83:834-838.

Hurley, R. 1990. Dwarf tall fescue: What to expect. Landscape Mgt. May 1990.

Iezzoni, A.F. and M.P. Pritts. 1991. Applications of principal component analysis to horticultural research. HortScience 26(4):334-338.

Meyer, W.A. and S.A. Bonos. 2001. Trends in cool-season turfgrass breeding. Golf Course Mgt. 69(9):61-64

Morris, K.N. 2001. National tall fescue test-1996. Final Rpt. 1997-2000. NTEP no. 01-14.
USDA-ARS, Beltsville, Md.

SAS Institute. 1985. In: The princomp procedure, $\mathrm{p}$ 621-637. SAS users guide: Statistics. vers. 5 ed. chapter 28. SAS Inst., Cary, N.C.

Shearman, R.C. and K.N. Morris. 1996. National tall fescue test-1992. Final report 1993-1995. NTEP no. 96-13. USDA-ARS, Beltsville, Md.

Watkins, E., W.A. Meyer, J.A. Murphy, S.A. Bonos, R.F. Bara, D.A. Smith, and W.K. Dickson. 2000. Performance of tall fescue cultivars and selections in New Jersey turf trials. Rutgers Turfgrass
Proc. 31:181-205.

Watkins, E., W.A. Meyer, J.A. Murphy, S.A. Bonos, M.M. Mohr, R.F. Bara, D.A. Smith, and W.K. Dickson. 2003. Performance of tall fescue cultivars and selections in New Jersey turfgrass trials. Rutgers Turfgrass Proc. 34:143-170.

Watkins, E., W.A. Meyer, J.A. Murphy, S.A. Bonos, M.M. Mohr, R.F. Bara, D.A Smith, and W.K. Dickson. 2002. Performance of tall fescue cultivars and selections in New Jersey turf trials. Rutgers Turfgrass Proc. 33:137-154. 